\title{
A High Resolution Optical Summary of the Structure and Dynamics of 30 Doradus
}

\author{
P. Scowen \\ Arizona State University, Physics \& Astronomy, P.O. Box 871504, \\ Tempe, AZ 85287-1504, USA \\ Y.-H. Chu, and R. Gruendl \\ Dept. of Astronomy, University of Illinois, Urbana, IL 61801, USA
}

\begin{abstract}
We present the first comparison between high resolution echelle velocity fields and a mosaic of all Hubble Space Telescope narrowband observations of 30 Doradus.
\end{abstract}

\section{Observations}

Our data include $3 \mathrm{HST}$ narrow-band image sets (WF/PC Hunter et al. 1995; WFPC-2 Scowen et al. 1998; WFPC-2 Chu et al. 1996) which together represent a large inner part of 30 Dor at a resolution of $0{ }^{\prime \prime} 1$ pixel $^{-1}$. The two WFPC-2 images have R136 cluster and Hodge 301 cluster centered in the PC, respectively. CTIO $4 \mathrm{~m}$ echelle observations were made to map the nebular kinematics in the R136 and Hodge 301 fields (marked by white rectangles in Fig. 1) The echelle observations have resolutions of $3.75 \mathrm{~km} \mathrm{~s}^{-1}$ and $0^{\prime \prime} 3$ pixel $^{-1}$. Iso-velocity maps are extracted and displayed in Fig. 2.

\section{Discussion}

Echelle iso-velocity maps reveal that gas around the R136 cluster appears blueshifted, while gas to the SE appears redshifted. If we assume Scowen et al.'s (1998) photoevaporative flow from the walls of the HII region (normal to the local interface), the observed velocities imply that gas around the cluster is on the back side of the nebula and gas to the SE of the cluster is on the near side of the nebula. The echelle data reveal large (10-20 pc diameter) rings and arcs that canot be seen in direct image, such as the ring south of R136. The echelle data also reveal high-velocity shocked gas that is not detected in direct images.

\section{References}

Chu, Y.-H., et al. 1996, BAAS, 188, 61.04

Hunter, D.A., et al. 1995, ApJ, 448, 179

Scowen, P.A., et al. 1998, AJ, 116, 163 


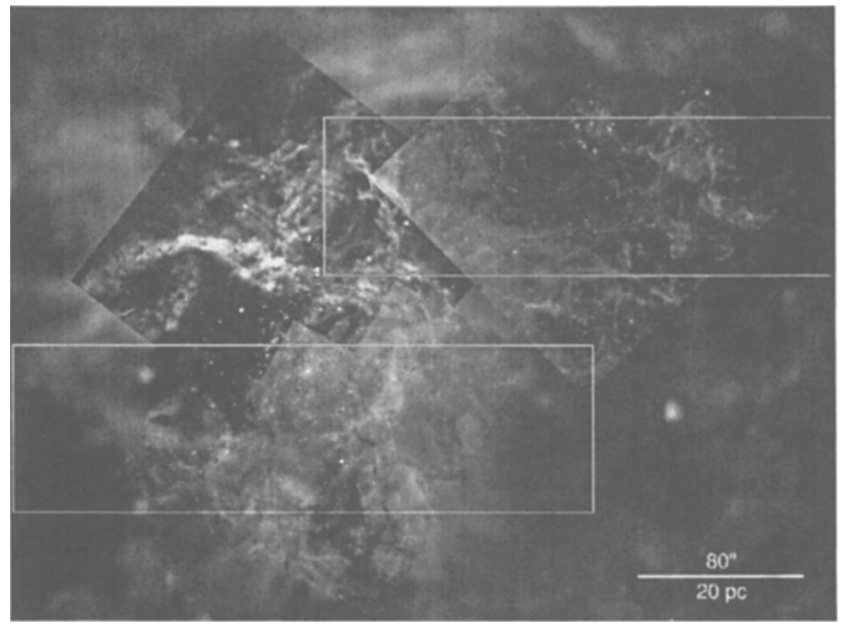

Figure 1. HST WFPC/WFPC2 images of 30 Dor. The two rectangles mark the areas, R136 and Hodge 301 fields, mapped by echelle observations.

Velocity Bin Size $=18.8 \mathrm{~km} / \mathrm{sec}$

Blue

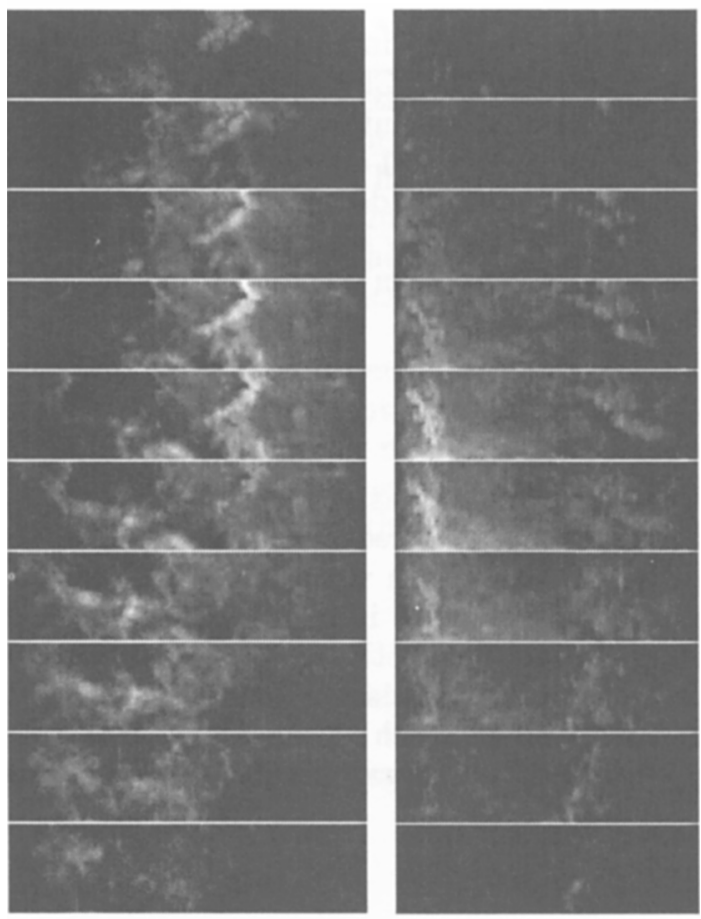

Red

Figure 2. Iso-velocity images extracted from the echelle mapping. The left column shows the R136 field and the right Hodge 301 field. 